\title{
Detectability of Rare Earth Elements in Explosive Post Blast Residues by Neutron Activation Analysis for use as Identification Taggants
}

Seman $\mathrm{J}^{1}$, Giraldo C.H. $\mathbf{C}^{2}$ and Johnson $\mathrm{C}^{2^{*}}$

${ }^{1}$ Department of Mining and Engineering, Missouri University of Science and Techology, USA

${ }^{2}$ Department of Mining and Nuclear Engineering, Missouri University of Science and Techology, USA

*Corresponding author: Dr. Catherine Johnson, Department of Mining and Nuclear Engineering, Missouri University of Science and Technology, USA, Telephone: +573-341-4258; Email: johnsonce@mst.edu

Received date: July 9, 2018; Accepted date: July 20, 2018; Published date: July 26, 2018

Copyright: $@ 2018$ Seman J, et al. This is an open-access article distributed under the terms of the Creative Commons Attribution License, which permits unrestricted use, distribution, and reproduction in any medium, provided the original author and source are credited.

\begin{abstract}
Current identification techniques can only identify the type of explosive used from traces in the post-blast residue. An identification taggant is a material added to an explosive that can provide identifying information about the explosive such as manufacturer, type, or date the explosive was produced. A novel identification taggant named the Nuclear Barcode is proposed, where specific elements in various concentrations allow up to 6.56 trillion different variations of the barcode. Holmium, samarium, and europium were chosen as taggant elements for initial testing purposes, the results of which are presented herein. Aqueous solutions and explosive post-blast residue where one or more taggant elements were added to the undetonated explosive were analyzed. Neutron activation analysis (NAA) was utilized to identify the elements present in each sample. A series of tests were completed in order to answer the question "can the Nuclear Barcode survive the explosion process and be adequately detected?" An identification taggant with this property is called a "survivable" taggant, this property is necessary for any identification taggant to be used as designed. Qualitatively, the results of the test series are successful, where taggant elements have been detected as much as $100 \%$ of tests performed. In tests where less than a $100 \%$ success rate occurred, it is theorized that it can be attributed to the delay between irradiating and counting being greater than the half-life of the element used. This method of tagging could prove useful for manufacturers of other products, since it has been proven to survive the high pressure and temperature detonation environment.
\end{abstract}

Keywords: Explosives taggants; Explosives; Rare earths; Forensic science; Explosives forensics; Taggants; Identification taggant; Explosives identification; Neutron activation analysis

\section{Introduction}

After a devastating terrorist attack or criminal bombing, one of the first questions on the investigator's mind is, "Where did the explosives come from?", in order to help find the culprit or prevent a repeat occurrence. In post-blast forensics, the answer to this question can be elusive [1-4]. Current investigative methods rely on finding undetonated components of the explosive device that was used, which allow law enforcement officials to identify the type of explosive used, initiation system or trigger source. Traces of the explosive may be found on suspects and matching those traces to those found at the blast site allows law enforcement to make a link [2,5]. Based on the magnitude of the blast, finding any components can be a challenge.

Investigating bombings where no traces of undetonated explosive are recovered requires the development and deployment of newer technologies such as identification taggants. Identification taggants are any substance that are added to an explosive that allow for the encoding of information about the explosive such as the manufacturer, type of explosive, or batch number of the explosive product. This technology has been investigated since the 1970s, with no ultimate solution being developed $[3,4]$. A tagant system can also be added to improvise explosive device precursors.

Four major eras in efforts to tag explosives in the United States for forensic investigations have been identified since the early $20^{\text {th }}$ century
[6]. These cycles have created the landscape of explosives laws and regulations existing today. Licenses for users of explosives began as a wartime measure in World War One to limit access to explosives to only those who had a need for it, and not enemy sympathizers or terrorists [7]. These measures were also revived in World War Two $[8,9]$. Passage of the Organized Crime Control Act in 1970 imposed these requirements permanently and went further by requiring identifying information, including the manufacturer, type of explosive, and when it was produced, to be written on the wrapper of all commercial explosives products [10]. The increased civil unrest that started the Organized Crime Control Act also prompted an investigation into more durable means of tagging explosives. Two laws requiring identification taggants to be included in all explosives were under consideration in 1978 and 1979 but were never signed into law [3,11]. The bombing in 1995 of the Murrah Building in Oklahoma City prompted the passage of the Effective Antiterrorism and Effective Death Penalty Act in 1996, which produced a report on identification taggants, but stopped short of recommending a legal requirement [4]. The September $11^{\text {th }}, 2001$ terrorist attack resulted in the passage of the Safe Explosives Act as a component of a larger bill that further restricted explosives licensing and began requirements for all explosives sales, not just interstate explosives sales [12]. With each successive cycle, proposed laws and regulations edged closer to requiring the use of some kind of identification taggant. Previous investigations into identification taggant technologies found them lacking in some evaluation area such as the cost, the ability of the identification taggant to survive an explosion, or the identification taggant altering the performance or sensitivity of the explosive $[3,4]$. 
Detonation of explosives produces high temperatures and pressures, which can break physical objects and cause chemical components to react. An identification taggant that can be successfully decoded or "read" once recovered after detonation is necessary. Identification taggants that have this trait are called "survivable". This paper describes new type of identification taggant called the Nuclear Barcode that has been decoded from post blast residues. A series of four questions for designing survivability tests of the Nuclear Barcode is presented. Based on these questions, a series of four tests were carried out and qualitative results are presented. The four test series were: single element standard solution tests, multi-element standard solution tests, single element post-blast test, and multi-element post-blast test.

\section{Nuclear Barcode Concept}

The nuclear barcode concept incorporates several rare elements to explosives at low concentrations, between 100 parts per billion (ppb) and $4000 \mathrm{ppb}$, during manufacturing that are detectable using neutron activation analysis (NAA) post blast. Each element would cover 40 concentration levels, each separated by $100 \mathrm{ppb}$ (Figure 1).

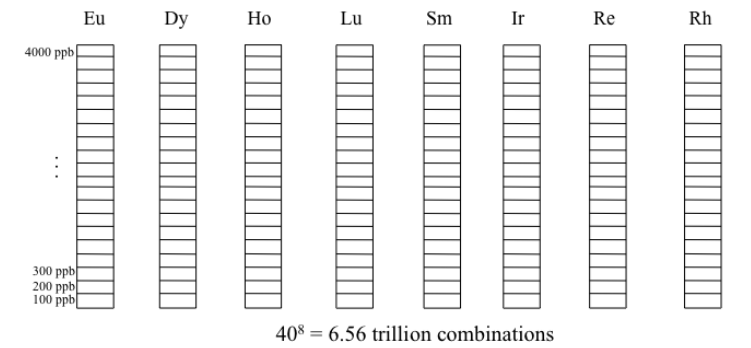

Figure 1: Schematic diagram of the nuclear barcode showing the concentration levels, elements used, and number of potential combinations.

NAA identifies elements by properties of their nuclei. To perform NAA, a sample is exposed to a neutron flux Different elements in the sample have a different probability of capturing a neutron and transforming into another isotope of the same element. This probability is the neutron capture cross section of the element, and different isotopes of the same element can have different neutron capture cross sections. Some of these isotopes will be radioactive and decay over time. When a radioactive nucleus decays, it emits a characteristic spectrum of gamma photons. When these photons encounter a detector, the energy spectra of the photons from all the decaying nuclei can be obtained and plotted. The energy spectra are plotted showing the number of photons the detector registers at each energy [13].

Using the nuclear barcode involves several steps. The first step is creating the identification taggant. For the nuclear barcode, the identification taggant is the unique combination of concentration levels of each of the eight elements used. This taggant is added to explosives during manufacturing in such a way that the taggant is well dispersed among the final product. When the explosive is detonated, the taggant elements remain behind in the solid phase as post-blast residue, where the taggant is recovered. Finally, post-blast samples are subjected to NAA. NAA allows the concentrations of the taggant elements to be determined, which gives the initial identification taggant. Figure 2 shows the cycle of creating, using, and reading of the nuclear barcode in an explosive:

To characterize the concept of survivability, four questions, with associated test series were posed:

- Can taggant elements be detected and quantified via NAA at the concentrations desired?

- Can different concentration levels of the taggant elements be distinguished and quantified via NAA?

- Can taggant elements be distinguished from the background, such as other taggant elements and other common elements in the environment such as sodium, potassium, chlorine, etc.?

- Can the concentrations of the taggant elements be quantified in the post blast residues and verified to be the same as in the undetonated sample?

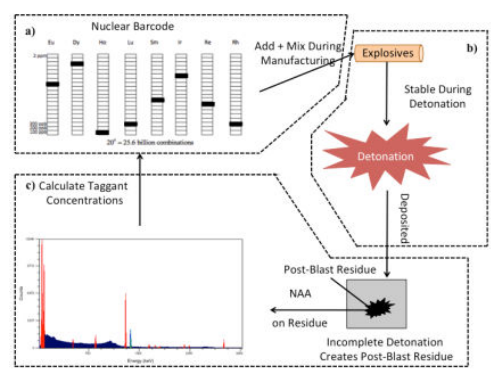

Figure 2: Creating (a), using (b), and reading the nuclear barcode (c) in an explosive.

\section{Experimental Design}

The results below focus on qualitative results using NAA, i.e. can the element(s) be individually identified, and not on the quantitative results where final concentrations of the elements are determined. Since the second and fourth questions are strictly quantitative, only the first and third questions will be addressed in this paper. The analysis of the quantitative results will be completed at a later date.

\section{Single element standard solution tests}

The nuclear barcode encodes information based on the concentration levels of the different component elements. Therefore, it is necessary to determine if the taggant elements can be identified at the low concentrations specified. Furthermore, for maximum utility, these elements must be able to be identified not only in undetonated explosive but also in the post-blast residue formed when a tagged explosive is detonated.

To address the first question regarding taggant detection at the concentration range of interest, solutions of three representative elements: holmium (Ho), samarium $(\mathrm{Sm})$, and Europium $(\mathrm{Eu})$ were tested. These three elements, individually, were dissolved in deionized (DI) water at five different concentration levels. The five concentrations used were $100,500,1000,2000$, and 4000 parts per 
Page 3 of 9

billion (ppb) on an atom basis. These concentrations cover the proposed range for the nuclear barcode. These tests were performed to evaluate the suitability of the candidate elements for analysis with NAA and the effectiveness of the chosen NAA parameters including neutron flux, irradiation time, counting time, and the delay time between irradiating and counting the sample. This experiment explores a bestcase scenario for detecting these elements: the test samples contain only the taggant element and DI water. Additionally, this test series can be looked at as a crude approximation of using the nuclear barcode in undetonated explosives. NAA is nearly insensitive to low atomic number elements such as carbon, hydrogen, oxygen, and nitrogen that make up common explosives such as ammonium nitrate fuel oil mixtures (ANFO), TNT, RDX, etc., so water, although chemically distinct, can serve as a crude simulant for undetonated explosives in which to detect the nuclear barcode [14].

Identifying the taggant elements is the first step towards calculating their concentrations. The authors have used an objective criterion for the purpose of identifying elements in the NAA spectrum. If the net number of counts underneath a peak (the difference between the area under the peak and the background) is greater than the calculated uncertainty in the measurement of the net number of counts under the peak, then the element corresponding to that peak was said to have been identified in the sample. Peaks were measured using Canberra's ProSpect ${ }^{\oplus}$ software. The results of performing NAA on five different concentrations of the holmium, samarium and europium are shown in Table 1, where the number of times the taggant element was detected is shown out of the number of tests run.

\begin{tabular}{|l|l|l|l|l|l|}
\hline Element & $\mathbf{1 0 0} \mathbf{p p b}$ & $\mathbf{5 0 0} \mathbf{p p b}$ & $\mathbf{1 0 0 0} \mathbf{p p b}$ & $\mathbf{2 0 0 0} \mathbf{p p b}$ & $\mathbf{4 0 0 0} \mathbf{p p b}$ \\
\hline Holmium & Yes-5/5 & Yes-5/5 & Yes-5/5 & Yes-5/5 & Yes-5/5 \\
\hline Samarium & Yes-5/5 & Yes $-4 / 4$ & Yes-5/5 & Yes-5/5 & Yes-5/5 \\
\hline Europium & Yes-5/5 & Yes-5/5 & Yes-5/5 & Yes-5/5 & Yes-5/5 \\
\hline \multicolumn{5}{|l}{ "One of the tests failed due to detector malfunction. } \\
\hline
\end{tabular}

Table 1: Number of completed tests where taggant element was detected in solution.

The taggant element was identified in all of the completed tests. One of the samarium $500 \mathrm{ppb}$ samples suffered a detector failure during counting, so data was not gathered for this sample. All other samples were counted for 1 hour. In each completed test, the taggant elements were correctly identified. This indicates that NAA sensitivity is sufficient to identify the tested taggant elements at the concentrations proposed for the nuclear barcode. Toxicology

Toxicological investigations were negative for alcohols and common drugs, also negative for anesthetics.

\section{Multi-element standard solution tests}

The multi-element standard solutions build on the single element standard solution tests by preparing samples that contained all three of the taggant elements in various concentrations. The presence of multiple elements produces more gammas, which could create either too much noise or overlapping peaks in the NAA spectrum and prevent accurately identifying the individual taggant elements. Six total combinations of the three elements at three different concentrations (500 ppb, $1000 \mathrm{ppb}$, and $2000 \mathrm{ppb}$ ) were prepared. For each mixture, five samples were prepared and tested. The different mixtures that were prepared, the concentrations of the three taggant elements used in each mixture, and the number of tests out of five where the elements were detected (Table 2).

\begin{tabular}{|l|l|l|l|}
\hline Mixture \# & Holmium & Samarium & Europium \\
\hline 1 & $500 \mathrm{ppb}(5)$ & $1000 \mathrm{ppb}(5)$ & $2000 \mathrm{ppb}(5)$ \\
\hline 2 & $500 \mathrm{ppb}(5)$ & $2000 \mathrm{ppb}(5)$ & $1000 \mathrm{ppb}(5)$ \\
\hline 3 & $1000 \mathrm{ppb}(5)$ & $500 \mathrm{ppb}(5)$ & $2000 \mathrm{ppb}(5)$ \\
\hline 4 & $1000 \mathrm{ppb}(5)$ & $2000 \mathrm{ppb}(5)$ & $500 \mathrm{ppb}(5)$ \\
\hline 5 & $2000 \mathrm{ppb}(5)$ & $500 \mathrm{ppb}(5)$ & $1000 \mathrm{ppb}(5)$ \\
\hline 6 & $2000 \mathrm{ppb}(5)$ & $1000 \mathrm{ppb}(5)$ & $500 \mathrm{ppb}(5)$ \\
\hline
\end{tabular}

Table 2: Multi Element Standard Solutions Table; number of tests out of 5 where taggant elements in multi-element standard solutions were detected for each combination in parentheses.

The taggant elements could be identified as separate elements in every test at these concentrations. This experiment indicates that these taggant elements will be able to be identified at all the concentration levels of the nuclear barcode. The multi-element standard solution series of tests was able to answer whether or not the three taggant elements would interfere with identifying one another.

A representative spectrum showing a sample of the low energy peaks created by each element (Figure 3), which shows that the peaks do not overlap and are easily identified.

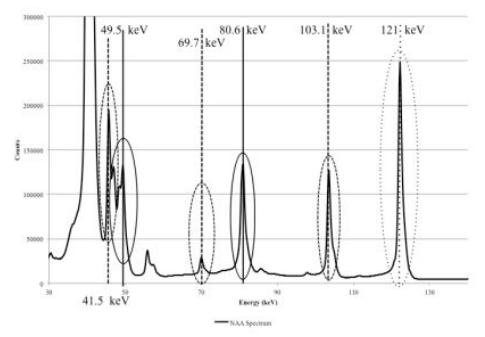

Figure 3: NAA spectrum of a representative multi-element standard solution containing $2000 \mathrm{ppb}$ holmium, $1000 \mathrm{ppb}$ samarium, and $500 \mathrm{ppb}$ europium. Peaks (circled) correspond to holmium (solid), samarium (dashed line), and europium (dotted line).

\section{Single element post-blast tests}

Charges of two types of explosives were prepared for this series of tests. The first sets of explosive charges were a commercially manufactured, cap sensitive binary explosive: ammonium nitrate and nitro-methane, which separately will not detonate, but when combined will detonate. The binary used has a maximum velocity of detonation (VOD) of $6,300 \mathrm{~m} / \mathrm{s}$ [15]. Five charges were prepared: two were tagged with holmium, two with samarium, and the fifth was untagged to provide a control. The second set of explosives charges were two 50 gram charges of cast Composition B manufactured on site and tagged with holmium. Composition $\mathrm{B}$ has a maximum VOD ranging from 7600 to $8000 \mathrm{~m} / \mathrm{s}$ [16]. Two tagged holmium charges were used to compare to the binary explosive charges tagged with holmium. The 
Page 4 of 9

tagged, undetonated explosive charges were put in a steel cylinder to provide a surface from which post-blast residue could be collected. A new steel cylinder was used for each test to eliminate crosscontamination between different tests. A steel cylinder acts as a stand in for a trashcan or a piece of debris where post-blast residue from a real terrorist attack might produce.

Due to the difficulties first encountered when measuring out quantities of taggant material on the small charges used in these experiments (160 grams for binary explosive charges down to 60 grams for composition B charges), larger concentrations than proposed for the nuclear barcode were added to the charges in this initial demonstration. For the holmium tests, the binary charges were each tagged with 0.00685 grams of holmium sulfate, giving a holmium mass concentration of $19000 \mathrm{ppb}$ to $20000 \mathrm{ppb}$ based on the manufacturing tolerances of the binary explosive. Similar concentrations were used for the samarium binary tests, but used 0.00789 grams of samarium sulfate per charge. For the holmium tagged composition B tests, 0.00170 grams of holmium sulfate was added giving a concentration of 12000 ppb to $13000 \mathrm{ppb}$. Terrorist attacks would use significantly higher amounts of explosive producing a larger amount of post blast residue. Once the tagging process is scaled up to a commercial manufacturing level, the whole process would be scaled up and the quantities of taggant materials for a typical batch of explosives would be easier to measure. Figure 4 shows the set-up of the charge within the cylinder (Figure $4 \mathrm{a}$ ) and the same cylinder after detonation (Figure $4 \mathrm{~b}$ ).
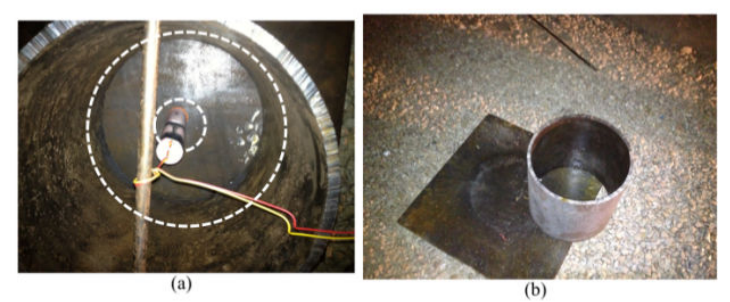

Figure 4: Single element post-blast test showing the commercially manufactured binary charge contained within the steel cylinder (a) and the same cylinder after detonation, with post-blast residue apparent at the center. Sampling path indicated by the dashed circles in Figure $4 \mathrm{a}$.

The testing methodology of all post-blast tests was based on the method developed for this series. After the charge was detonated, samples of the post-blast residue were obtained using a cotton ball and sampling around the middle of the cylinder and the bottom plate [14]. These tests also establish if the taggant elements will encounter any interference from the other elements present in the post blast residue. The presence or absence of an element in the post-blast residue can be determined by the presence or absence of a peak at the characteristic energy corresponding to the element. Results for the post-blast tests using the different tagged binary charge (Table 3) [6].

\begin{tabular}{|l|l|l|l|}
\hline Test & $\begin{array}{l}\text { Taggant } \\
\text { Added }\end{array}$ & $\begin{array}{l}\text { Holmium } \\
\text { Counts } \\
\mathbf{8 0 . 6} \mathbf{~ k e V} \text { peak) }\end{array}$ & $\begin{array}{l}\text { Net } \\
\text { Samarium } \\
\text { (ounts } \\
\mathbf{6 9 . 6} \mathbf{~ k e V ~ p e a k )}\end{array}$ \\
\hline 1 & Holmium & $2,236 \pm 668$ & $290 \pm 1,306$ \\
\hline 2 & Holmium & $98,026 \pm 1,744$ & - \\
\hline 3 & Samarium & $63 \pm 1,490$ & $10,872 \pm 1,299$ \\
\hline 4 & Samarium & $3,372 \pm 1,762$ & $66,381 \pm 2,589$ \\
\hline 5 & Control & $766 \pm 1,802$ & $849 \pm 988$ \\
\hline
\end{tabular}

Table 3: Taggant elements present, net counts, and uncertainties of holmium and samarium tagged binary post-blast residues, with elements detected in the samples bolded.

For tests one and two, holmium was added to the undetonated explosive. In both of these tests, the net number of counts under the peak is greater than the uncertainty in the number of counts, and therefore holmium was found to be present in these tests. The results for samarium in these tests are shown as well, and indicate that samarium was not found in the first test, and a measurement of the peak in the second test was not able to be made due to a technical error where the software used failed to measure the area under the peak [6].

For tests three and four, samarium was added to the undetonated explosive. In both of these tests, the number of net counts under the peak is once again larger than the uncertainty in the number of counts, indicating that samarium was present in the samples. Test three shows no presence of holmium in the sample. Test four shows a spurious holmium presence, as an additional peak near, but not at, the energy corresponding to holmium and can be disregarded [6].

The series of tests performed using a Composition B focused on identifying the taggant element holmium, when added to the undetonated explosive in the post-blast residue. Since post-blast residues contain many elements, the primary concern is determining if the taggant elements can be found. Results for the post-blast tests using a tagged composition B charge (Table 4).

\begin{tabular}{|l|l|l|}
\hline Test & Taggant Element & $\begin{array}{l}\text { Holmium } \\
\text { Counts }\end{array}$ \\
\hline 1 & Holmium & $54523 \pm 820$ \\
\hline 2 & Holmium & $5384 \pm 42206^{*}$ \\
\hline $\begin{array}{l}\text { *Denotes that although this test fails the objective criterion used, further analysis } \\
\text { shows that holmium is present in this test sample. }\end{array}$ \\
\hline
\end{tabular}

Table 4: Taggant elements present, net counts, and uncertainties of holmium tagged composition B post-blast residue (elements detected in the samples bolded).

Test 1 clearly shows the presence of holmium in the post-blast residue. Test 2 does not, despite being tagged with holmium before detonation. However, on inspecting the NAA spectrum from Test 2, which is shown in Figure 5, there is a clear peak at the $80.6 \mathrm{keV}$ that is expected for holmium. 


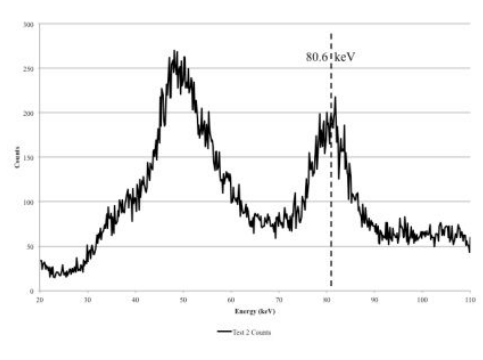

Figure 5: NAA spectrum from test 2 of single element composition $\mathrm{B}$ post-blast series showing the presence of holmium with peak at $80.6 \mathrm{keV}$ indicated by the dashed vertical line.

The asterisk in the table denotes that although the number of net counts, from the software, does not show the presence of the taggant element, the spectrum convincingly does. In other words, we conclude that while the software can be helpful, it is sometimes necessary to manually check the results to confirm the software's conclusions. On the other hand, the goal of this series of tests was to determine if the taggant elements can be identified in the post-blast residue. In each of the six tests where a taggant element was added to an explosive, the taggant element could successfully be detected.

\section{Multi element post-blast tests}

The multi-element binary post-blast test series adds the complication of recovering three taggant elements in the post-blast residue. A total of six combinations of the three elements were each added to an undetonated explosive; the concentrations added were $1000 \mathrm{ppb}, 2000 \mathrm{ppb}$, or $4000 \mathrm{ppb}$ to determine if the taggant elements can be identified at concentrations used by the nuclear barcode. Each element was tested twice at these concentrations. This series of tests essentially used a cut down version of the nuclear barcode with fewer total combinations.

This represents a real world test on detecting the taggant elements and determining their concentrations from the post-blast residue using concentrations proposed by the nuclear barcode. The results from this series of tests indicate that the taggant elements can be recovered in the post-blast residue simultaneous. Further testing is necessary to determine the minimum concentration that can realistically be used, this however might require an improved manufacturing process that can reliably dose the quantities of taggant material needed for concentrations below $1000 \mathrm{ppb}$ with the charge size used.

A representative binary charge (test 3 on Table 8 below) was tagged with 0.00099 grams of holmium sulfate, 0.00203 grams of samarium sulfate, and 0.00049 grams of europium sulfate. This resulted in concentrations of $2600 \mathrm{ppb}$ of holmium, $5100 \mathrm{ppb}$ samarium, and 1200 ppb europium by mass. Tagged explosives were placed within a steel cylinder to provide a surface to collect the post-blast residue. Figure 6 shows the experimental configuration of the tagged explosive in the steel cylinder before detonation (Figure 6a) and the deposited postblast residue after detonation (Figure $6 \mathrm{~b}$ ).

Figure 6: Multi-Element binary post-blast charge and witness plate before detonation (a) and deposited post-blast residue after detonation (b).

Additional tests were designed to determine the repeatability of using the nuclear barcode. The first of these charges used the same commercially available binary explosive as used in the single element post-blast test, and the second used $100 \mathrm{~g}$ cast composition B charges that were manufactured on site. These charges were then tagged with one particular combination of the three taggant elements: 2000 ppb of holmium, $1000 \mathrm{ppb}$ of samarium, and $4000 \mathrm{ppb}$ of europium. All of the multi element post blast tests are summarized in Table 5 .

\begin{tabular}{|l|l|}
\hline Test Series & Number of Samples \\
\hline Multi Element Binary Post-Blast & 6 \\
\hline Multi Element Composition B Post-Blast & 6 \\
\hline Multi Element Binary Repeatability & 5 \\
\hline Multi Element Composition B Repeatability & 5 \\
\hline
\end{tabular}

Table 5: Multi Element Post-Blast Tests Summary.

As with the single element post-blast tests, the presence and absence of elements can be assessed by determining the net number of counts under the peaks, and the uncertainties in the net counts, corresponding to that element in the NAA spectrum obtained from the sample. The net counts and the uncertainties for each of the three taggant elements added to a binary charge are shown in Table 6.

\begin{tabular}{|l|l|l|l|l|l|l|}
\hline Test & Holmium Net Counts & Holmium Present & Samarium Net Counts & Samarium Present & Europium Net Counts & Europium Present \\
\hline 1 & $377.27 \pm 172.55$ & Yes & $744.72 \pm 294.59$ & Yes & 0 & No \\
\hline 2 & $551.95 \pm 273.78$ & Yes & 0 & No & 0 & No \\
\hline 3 & $147.09 \pm 325.00$ & No & $2383.53 \pm 359.49$ & Yes & 0 & No \\
\hline 4 & $122.32 \pm 194.84$ & No & 0 & No & 0 & No \\
\hline
\end{tabular}


Page 6 of 9

\begin{tabular}{|l|l|l|l|l|l|l|}
\hline 5 & $216.11 \pm 200.87$ & Yes & $180.80 \pm 243.56$ & No & 0 & No \\
\hline 6 & $118.49 \pm 148.10$ & No & $774.50 \pm 163.65$ & Yes & 0 & No \\
\hline
\end{tabular}

Table 6: Taggant elements present, net counts, and uncertainties in multi-element binary post-blast residues (elements detected in the samples bolded).

In each of these tests, europium could not be identified. There are two possible reasons for this. Europium has two stable, naturally occurring isotopes, 151Eu and 153Eu [17]. During NAA, these elements produce isotopes that have relatively long half-lives on the order of several years. This long half-life, however, makes these elements relatively inactive, and thus they require longer counting times, making their use not feasible for this experiment. Activation also produces the europium isotope $152 \mathrm{mlEu}$, a metastable isotope with a half-life of 9.29 hours [17].

This more active isotope is the one that has been used to specifically identify the presence of europium. The post-blast residue sample from the first test in this series began counting 70 hours after irradiation. This delay was necessary to allow safe handling of the samples due to the activity of some shorter lived isotopes including manganese, silicon, and aluminium that were picked up from the environment. This period of time is approximately 7.5 half-lives, so the amount of the active isotope being measured has been reduced by a factor of 27.5 , or approximately $1 / 186^{\text {th }}$ of its original concentration. Therefore, the activated europium allows for a considerably shorter counting time, but it may be too unstable to be seen after the required delay period.

The second possible reason for the absence of europium in the postblast samples is that none of the added europium taggant was collected. While, the elements added to the explosive cannot be destroyed during the detonation process, it could be that the europium partitions in the cloud of particulate material generated by the detonating explosive differently than holmium and samarium.

This possibility will need further study. However, since all three elements are rare earth elements, they should be chemically similar, which suggests they should act similarly during detonation. While the data collected cannot discriminate between the two possible reasons, we consider it more likely that the time delay between irradiating the samples and counting them is responsible for the lack of any measureable quantity of europium in the samples.

The results for holmium are positive. All samples showed traces of holmium, however tests three, four, and six did not show more net counts than the uncertainty in the measurement. A peak at the correct energy for holmium was observable for each test, similar to the results for test two of the single element composition B test series, but the measured net counts do not meet our criteria for stating that holmium is present in those tests.

The results for samarium are mixed. In two samples, no trace of a samarium peak at $69.6 \mathrm{keV}$ was found. From the single and multielement standard solutions tests, this peak was found to be the most specific peak indicating the presence of samarium, despite larger peaks present at 41.5 and $103.1 \mathrm{keV}$ which had other peaks close enough to interfere with measurement. Of the remaining tests, three showed net counts well in excess of the uncertainty, and one does not meet that criteria. The result from test five, like the holmium results from tests three, four, and six; have noticeable peaks, but does not meet the criterion to say that samarium is present.

In the end, this test did not provide a clear answer to determining if the taggant elements interfere with one another. In all the tests where the taggant elements can be shown to be present, their peaks are distinct from those from other elements present in the sample. However, due to the presence of other environmental material, the post-blast residue becomes highly active when irradiated under the test conditions, and a period of time is needed to allow the samples to "cool" sufficiently so that they can be handled safely. This period of time was too long for the 9.29 hour half-life of metastable 152Eu isotope. More tests will need to be performed. Both holmium and samarium are recommended as successful candidates for the nuclear barcode and with the increased knowledge from this study, additional taggant options will be selected with similar half-lives for ease of reading the barcode post detonation.

The series of tests on tagged Composition B uses the same combination of three elements at three concentration levels to create six unique combinations of taggants that was used in the multielement binary post-blast test series described previously. Comparing the number of net counts to the uncertainty in the net counts provides a good method of identifying if the taggant element is present in the sample (Table 7).

\begin{tabular}{|l|l|l|l|l|l|l|}
\hline Test & Holmium Net Counts & Holmium Present & Samarium Net Counts & Samarium Present & Europium Net Counts & Europium Present \\
\hline 1 & $91.107 \pm 236.34$ & No & $124.90 \pm 211.64$ & No & 0 & No \\
\hline 2 & $312.68 \pm 198.07$ & No & $705.81 \pm 259.58$ & Yes & $96.14 \pm 235.48$ & No \\
\hline 3 & $99.40 \pm 351.30$ & No & $445.28 \pm 183.78$ & Yes & 0 & No \\
\hline 4 & $352.03 \pm 203.59$ & Yes & $1466.35 \pm 254.15$ & Yes & $141.91 \pm 529.62$ & No \\
\hline 5 & 0 & No & $223.20 \pm 159.70$ & Yes & 0 & No \\
\hline
\end{tabular}


Page 7 of 9

\begin{tabular}{|l|l|l|l|l|l|l|}
\hline 6 & $91.66 \pm 96.99$ & No & $537.86 \pm 208.04$ & Yes & 0 & No \\
\hline
\end{tabular}

Table 7: Taggant elements present, net counts, and uncertainties in multi-element Composition B post-blast residues (elements detected in the samples bolded).

Results are similar to the binary post-blast tests. Samarium and holmium can be found in most tests, while europium cannot be definitively located. Unlike the tests on binary post-blast samples, europium peaks can be noticed in the spectra of some tests, but not enough counts are available for adequate quantification. The amount of europium measured here is small and close to being considered "not detected". These samples were counted approximately 20 hours sooner than the samples from the mixed element binary post-blast test series. This additional data supports the suggestion that the problem locating europium is radioactive decay.

The peaks of the three taggant elements are all well separated from the peaks coming from other elements present in the sample. However, not all samples showed peaks for all three taggant elements. This partially answers the question of if the taggant elements interfere with one another or are lost in the background. Taggant peaks are located at distinct energies from the background, but the peaks can be lost due to the time needed to allow the samples to be handled after activation.

The two sets of tests on the repeatability of post-blast residues provide a way of analyzing the differences between the types of explosives and the recoverability of some taggant elements that was observed with the commercial binary and Composition B explosive charges. Additionally, variation between tests is expected, so by using identical charges, information about the repeatability of the nuclear barcode can be obtained. The results of analyzing the five identically tagged binary charges (Table 8).

\begin{tabular}{|l|l|l|l|l|l|l|}
\hline Test & Holmium Net Counts & Holmium Present & Samarium Net Counts & Samarium Present & Europium Net Counts & Europium Present \\
\hline 1 & $538.71 \pm 197.90$ & Yes & $1815.94 \pm 389.52$ & Yes & $521.29 \pm 280.97$ & Yes \\
\hline 2 & $394.81 \pm 170.12$ & Yes & $290.30 \pm 120.63$ & Yes & $227.79 \pm 291.55$ & No \\
\hline 3 & $2709.14 \pm 224.31$ & Yes & $1162.77 \pm 226.54$ & Yes & $286.42 \pm 250.78$ & Yes \\
\hline 4 & $176.38 \pm 484.80$ & No & $1381.30 \pm 204.16$ & Yes & $201.56 \pm 272.21$ & No \\
\hline 5 & $382.73 \pm 244.62$ & Yes & $1773.01 \pm 255.60$ & Yes & $439.94 \pm 299.55$ & Yes \\
\hline
\end{tabular}

Table 8: Taggant elements present, net counts, and uncertainties in multi-element binary repeatability post-blast residues (elements detected in the samples bolded).

There were three instances where an element was not found in the post-blast residue: europium in the second test, and both holmium and europium in the fourth test. The advantage of this series of tests is the same concentrations of taggant elements were added to undetonated explosive.

Despite the noisy environment produced during detonation, the taggant elements could be identified in 12 of 15 cases in this series of tests. Additional elements in the sample produce their own gammas and due to scattering and reflection produce extra background in the NAA spectrum. This is a normal process and happens with all samples that undergo NAA, however searching for trace elements such as the taggant elements used in the nuclear barcode exacerbates the issue.

This process of losing energy produces a broad spectrum of photons from the narrow peaks emitted by a radioactive species, and multiple species all increase this broad spectrum noise. The ones where the taggant elements could not be positively identified were because number of net counts was lower than the uncertainty. However, in those cases, the peak was still there, but very small.

The second set of repeatability tests in Composition B charges also provides additional information about the repeatability of the nuclear barcode. To show the detectability of these elements, the net counts and the uncertainties in the net counts that were obtained from the five identically tagged Composition B charges (Table 9).

\begin{tabular}{|c|c|c|c|c|c|c|}
\hline Test & $\begin{array}{l}\text { Holmium } \\
\text { Net } \\
\text { Counts }\end{array}$ & $\begin{array}{l}\text { Holmiu } \\
\text { m } \\
\text { Present }\end{array}$ & $\begin{array}{l}\text { Samarium } \\
\text { Net } \\
\text { Counts }\end{array}$ & $\begin{array}{l}\text { Samariu } \\
\text { m } \\
\text { Present }\end{array}$ & $\begin{array}{l}\text { Europiu } \\
\text { m Net } \\
\text { Counts }\end{array}$ & $\begin{array}{l}\text { Europiu } \\
\text { m } \\
\text { Present }\end{array}$ \\
\hline 1 & $\begin{array}{l}197.37 \quad \pm \\
97.90\end{array}$ & Yes & $\begin{array}{l}30.65 \\
631.94\end{array}$ & No & 0 & No \\
\hline 2 & 0 & No & $\begin{array}{l}328.98 \\
155.69\end{array}$ & Yes & 0 & No \\
\hline 3 & $\begin{array}{l}231.31 \\
217.42\end{array}$ & Yes & $\begin{array}{l}110.01 \\
156.95\end{array}$ & No & 0 & No \\
\hline 4 & $\begin{array}{l}165.45 \\
207.91\end{array}$ & No & $\begin{array}{l}126.14 \quad \pm \\
89.52\end{array}$ & Yes & 0 & No \\
\hline 5 & $\begin{array}{l}138.68 \\
161.62\end{array}$ & No & 0 & No & 0 & No \\
\hline
\end{tabular}

Table 9: Taggant elements present, net counts, and uncertainties in multi-element composition B repeatability post-blast residues (elements detected in the samples bolded).

Peaks corresponding to the holmium and samarium can be seen in all but one test each, however these elements can only be definitively said to be present in a couple tests. Holmium can only said to be present in test three, while samarium can only said to be present in tests two and four. Once again, the time between irradiation and counting appears to have allowed for any activated europium to decay 
away. The composition B repeatability post-blast residue tests had a roughly $12.5 \%$ higher delay time (54 hours vs 48 hours) between irradiating the sample and counting them compared to the binary repeatability post-blast residue tests. This additional time could account for the difference in the detectability of europium in the two test series.

As with previous samples, the taggant element peaks can be readily distinguished from peaks resulting from other background elements present in the sample. However, not all samples showed notable peaks for the taggant elements. Taking additional samples of the post-blast residue would provide an additional data points to determine if the elements are truly not present in the post-blast residue for those tests, or if they were not identifiable in that particular sample of the postblast residue.

\section{Conclusions}

This paper has set forth four questions to answer and assess the survivability of the nuclear barcode as an identification taggant and provides qualitative answers for two of them:

- Can taggant elements be detected and quantified via NAA at the concentrations desired?

- Can different concentration levels of the taggant elements be distinguished and quantified via NAA?

- Can taggant elements be distinguished from the background, such as other taggant elements and other common elements in the environment such as sodium, potassium, chlorine, etc.?

- Can the concentrations of the taggant elements be quantified in the post blast residues and verified to be the same as in the undetonated sample?

Qualitatively, the taggant elements tested can be identified in most circumstances, though difficulties exist and complicate or prevent identification in some samples. Most often, europium was unable to be identified in post-blast samples thought to be due to the short half-life of the isotope used in this analysis. Other isotopes of europium are also active under NAA, and might be better suited than the $151 \mathrm{~m} 1 \mathrm{Eu}$ isotope used. Alternatively, if the other isotopes of europium prove to be intractable, then europium is not suitable for the Nuclear Barcode. Quantitative analysis of these samples is on-going, and allowing us to further understand the suitability of the Nuclear Barcode as an identification taggant.

The first question posed asks if the taggant elements can be identified at the concentrations the nuclear barcode is designed to use. Solutions of single and multiple taggant elements dissolved in water covering the entire concentration range of the nuclear barcode were prepared and analyzed using NAA. In every case where the test was successfully completed, every taggant element was identified. Therefore, under ideal circumstances, the answer is a definitive yes. These tests can be considered a simulated use of the nuclear barcode in an undetonated explosive, so this indicates that the nuclear barcode is of potential use for identifying explosives before detonation. The tests of post-blast residue are less definitive than the ideal case, but it should be noted that the extreme environment of a detonation is a worst case scenario for a taggant and this system could easily be used in other processes. In tests of post-blast residue of charges that were tagged with one element, the taggant element used could be identified in each test. In tests where multi-element tagged charges were used, the analysis of results became more difficult. In most of these, the longer lived, activated isotopes of holmium and samarium were identified, but the shorter-lived isotope of Eu-151m2 that was used to identify europium was usually not found. The relatively short half-life of this europium isotope, compared to the time between irradiating the sample and counting the sample is the major cause for the inability to identify europium in the post-blast samples.

The third question asks if the different taggant elements can be identified over the background noise in the NAA spectrum. This question can be broken down into two parts: can the different taggant elements be distinguished from one another, and can the different taggant elements be distinguished from the other elements present in the sample. The former case is answered affirmatively by the success in identifying the individual taggant elements in multi-element solution test. In each sample, the taggant elements were able to be resolved simultaneously. The latter case is answered affirmatively by the postblast test series. Although many elements were identified in the postblast residue NAA spectrum, it was always the case that the location of a peak corresponding to the taggant elements was able to be found. This result means that in the cases where the taggant elements were found, a distinct peak was located at a signature energy corresponding to that taggant element. This result also means that in the cases where no taggant element was found, the spectrum was locally flat. Therefore it was concluded that the element cannot be identified because the element is not present due to either not being included in the tag used for that particular charge, or because the isotope of the element used in NAA decayed too quickly to be identified.

Combined, these results suggest that the Nuclear Barcode has potential. Holmium, samarium, and europium in water can be measured at as low a concentration as $100 \mathrm{ppb}$. The same elements can be identified in post-blast residue at pre-detonation concentrations of approximately $1000 \mathrm{ppb}$, although europium might be too short lived for future use. Using concentrations this low for the Nuclear Barcode reduces the price of adding the expensive taggant materials to a reasonable level necessary to be widely used.

\section{Future Work}

The signal to noise ratio when performing NAA on post-blast samples containing several elements needs to be addressed. To identify an element, the peaks corresponding to that element (the signal) must be able to be differentiated from the background from all the other gammas that are being emitted at the same time (the noise). The more counts under the peak but over the background, the higher quality the NAA results.

Obtaining high quality NAA results depends on several parameters that need to be optimized. The time between irradiating and counting is too long compared to some of the half-lives of the isotopes produced. This time delay then means the signal that is being searched for decays away before it can be counted and thus an element that is present cannot be identified. Optimizing the time delay between irradiating and counting the samples is therefore one method of increasing the signal to noise ratio obtained via NAA.

Quantitative measurements of the concentrations of the elements identified by the tests described in previous sections are underway and will enable for a fuller determination of the viability of the nuclear barcode as an identification taggant. These quantitative measurements are addressed by the second and fourth questions that were used to create this series of tests. A viable version of the nuclear barcode requires that concentrations be measurable to a high degree of precision so that the concentration can be determined to be within on 
Citation: Seman J, Giraldo C.H.C, Johnson C (2018) Detectability of Rare Earth Elements in Explosive Post Blast Residues by Neutron Activation Analysis for use as Identification Taggants. J Forensic Res 9: 423. doi:10.4172/2157-7145.1000423

Page 9 of 9

$100 \mathrm{ppb}$ concentration level. This degree of precision requires a sufficiently high signal to noise ratio from the NAA spectrum that the standard deviation in the measured concentration is less than $25 \mathrm{ppb}$. Additionally, this degree of precision requires that the NAA measurements are reproducible, and that the inter-sample variance of the concentrations determined by NAA is also smaller than $25 \mathrm{ppb}$. While well studied in many fields, the use of NAA on explosive postblast residue is new and the ability of NAA to perform to this required degree of precision requires substantial testing and analysis that is ongoing but current results indicate great promise.

\section{References}

1. Abdul-Karim N, Blackman CS, Gill PP, Wingstedt EMM and Reif BAP (2014) Post-blast explosive residue - A review of formation and dispersion theories and experimental research. RSC Adv 4: 54354-543721.

2. Hoffman CM and Byall EB (1974) identification of explosive residues in bomb scene investigations. J Forensic Sci 19: 54-63.

3. Office of Techology Assesment - Congress of the United States (1980) Taggants in Explosives Congress of the United States, Washington DC, USA.

4. Committee on Marking, Rendering Inert, and Licensing of Explosive Materials (1998) Containing the threat from illegal bombings an integrated national strategy for marking, tagging, rendering inert, and licensing explosives and their precursors. Board on Chemical Sciences and Technology Commission on Physical Sciences, Mathematics, and Applications, National Research Council, Washington DC, USA.

5. Harvey JM Hou (2013) Toward chemical signature analysis of forensic evidences. J Forensic Res 4 :e113.

6. Seman J, Johnson C, Giraldo C, (2017) Holmium and samarium detectability in post-blast residue. in proceedings from the 20th biennial international conference of the aps topical group on shock compression of condensed matter, USA.

7. Munroe CE (1921) Regulation of explosives in the united states with especial reference to the administration of the explosives act of 1917. Department of Interior, Bureau of Mines, Washington DC, USA.

8. Sayers RR (1940) General information and rulings for the enforcement of the law regulating the manufacture, distribution, storage, use, or possession of explosives and their ingredients during wartime. Department of the Interior, Bureau of Mines, Washington DC, USA.

9. Sayers RR (1940) Federal explosives act of December 26, 1941 (55 stat. 863), as amended regulations issued under the federal explosives act recommendations for storing, handling and transporting explosives. Department of the Interior, Bureau of Mines, Washington DC, USA.

10. Your guide to explosives regulation (1976) Bureau of Alcohol, Tobacco, and Firearms, USA

11. Committee on the Judiciary (1975) State Department Bombing by Weatherman Underground Hearing Before the Subcommittee to Investigate the Administration of the Internal Security Act and Other Internal Security Laws of the Committee on the Judiciary United States Senate Ninety-Fourth Congress, USA.

12. Safe Explosives Act (2002), Public Law No. 107-296, 116 Stat. 2135, USA.

13. Lamarsh JR, Baratta AJ (2001) Introduction to Nuclear Engineering Prentice Hall, USA.

14. Tourné M, (2013) Developments in Explosives Characterization and Detection. J Forensic Res 4:S12.

15. Technical Data Sheet Kinepak (2009) Hallowell Manufacturing LLC, USA.

16. Cooper WP (1996) Explosives Engineering. Wiley publication, USA.

17. Chart of the Nuclides 10 Knolls Atomic Power Laboratory, USA. 